\title{
Segmental Facial Nerve Decompression in Patients of Chronic Suppurative Otitis Media with Diabetes Mellitus
}

\section{Rathod J, Karkamkar $S^{*}$ and Dongardive S}

Department of ENT, Mahatma Gandhi Mission Medical College, India

*Corresponding author: Shradha Karkamkar, Department of ENT, Mahatma Gandhi Mission Medical College and Hospital, Aurangabad, India, Tel: 09075710970; Email: shradhajk123@ gmail.com

\section{Research Article \\ Volume 6 Issue 1}

Received Date: January 25, 2021

Published Date: February 12, 2021

DOI: $10.23880 /$ ooaj-16000207

\section{Abstract}

Introduction: Facial nerve decompression is opening the bony canal and nerve sheath to release pressure and reduce compression on nerve fibers. The goal of decompression is to improve blood circulation and minimize damage to distal nerve fibers.

Methodology: We present study of 20 patients who came to ENT OPD, From June, 2017 -March, 2020 presented to us with non-traumatic facial palsy grade III and above; in whom we did facial nerve decompression via trans-mastoid approach.

Conclusion: Segmental decompression of facial nerve with masoidectomy provides better results than mastoidectomy alone because it enables expansion of nerve, reduces post-operative oedema and less need for steroid administration in diabetic patients with better clinical outcome.

Keywords: Facial paralysis; Diabetes mellitus; Decompression; Trans-mastoid approach; Tympanic segment; 1st genu; Stylomastoid foramen; Bony canal

Abbreviations: HB classification: House Brackmann classification; CSOM: Chronic Suppurative Otitis Media.

\section{Introduction}

Decompression means opening of bony canal and nerve sheath to release the pressure and decrease compression over the nerve fibers. The goal of decompression is to improve blood circulation and minimize damage to distal nerve fibers. The facial nerve is the largest nerve that travels in bony canal with a complex course with a high susceptibility to injury. Lack of space for expansion in a rigid bony canal, potentially leads to severe nerve damage and even necrosis and fibrosis. Facial palsy causes significant functional and cosmetic defects that mostly leads to great psychological impact. The primary goal of management in patients with facial paralysis, whichever may be the etiology is to increase functional recovery and minimize the cosmetic defect. When complete paralysis is either due to anatomic discontinuity or neural degeneration, the facial nerve requires repair or decompression for the optimum functional and aesthetic results. Whether the facial nerve decompression has to be done or not is really a surgeon's dilemma. Whether the patient will be benefited if the pressure on facial nerve is released or not is the real big question. Few surgeons and authors recommend that only mastoidectomy and removal of cholesteatoma or granulation tissue will be enough and facial nerve function might recover after the removal of etiology. We selectively decompressed the involved facial nerve segment along with few millimeters proximal and distal to the lesion (which is not similar to the conventional method where the subtotal nerve right from 1st genu to stylomastoid foramen is decompressed). Some authors have noted to decompress the labyrinthine and meatal segments 
of the facial nerve only. These recommendations were based on studies showing that probably these two segments are the narrowest portions of the temporal bone at which nerve conduction blockage occurs most of the times. Some have insisted subtotal decompression from the labyrinthine segment to the stylomastoid foramen based on their results showing reduced recurrences of facial palsy and improved recovery rate. Regarding timing of surgery, it is generally accepted that most favorable outcomes are obtained when decompression is performed at the earliest after onset of symptoms .While some studies suggest potential benefits from delayed decompression anywhere from 1 month up to 4 months following the onset of the paralysis.

\section{Aims and Objectives}

To study the need of facial nerve decompression surgery in patients of facial palsy to preserve and restore facial nerve function.

\section{Materials and Methods}

It is a retrospective study of need and subsequent improvement following facial nerve decompression surgery in patients with Diabetes. We present study of 20 patients who came to ENT OPD, From June, 2017 -March 2020, presented to us with ear discharge \& facial palsy grade III and above; in whom we did facial nerve decompression through trans-mastoid approach. The inclusion criteria were patient's age more than 30 years, who gave informed consent and had secondary facial nerve palsy having acute onset of symptoms and having diabetes mellitus. The exclusion criteria were those who did not give informed consent and who had history trauma which was the cause of facial nerve palsy and having similar complains in past. Data was collected using a structured questionnaire. The data covered background information about the participant and a detailed history regarding chief complaints associated symptoms, past, family and personal history. Basic general and systemic examination was done. Local examination of the ear and detailed examination of 7 th cranial nerve was done. Examination of other cranial nerves and ENT examination was also included in our study. Routine blood and audiological investigations like PTA and acaustic reflex test and radiological investigations like HRCT temporal bone were given emphasis. Facial nerve decompression was done by surgical intervention via trans-mastoid approach. Assessment of results was done again by House- Brackmann grading system and improvement to Grade II- I of facial nerve function was considered as successful outcome. The procedure performed was in accordance with Ethical committee on human experimentation and with Helsinki declaration of 1975 revised on 2000. Data was entered in
Microsoft excel and results were calculated in the form of frequency, percentages and proportions, chi square test were applied to qualitative data using software SPSS trial version 20 (Figures 1-5).

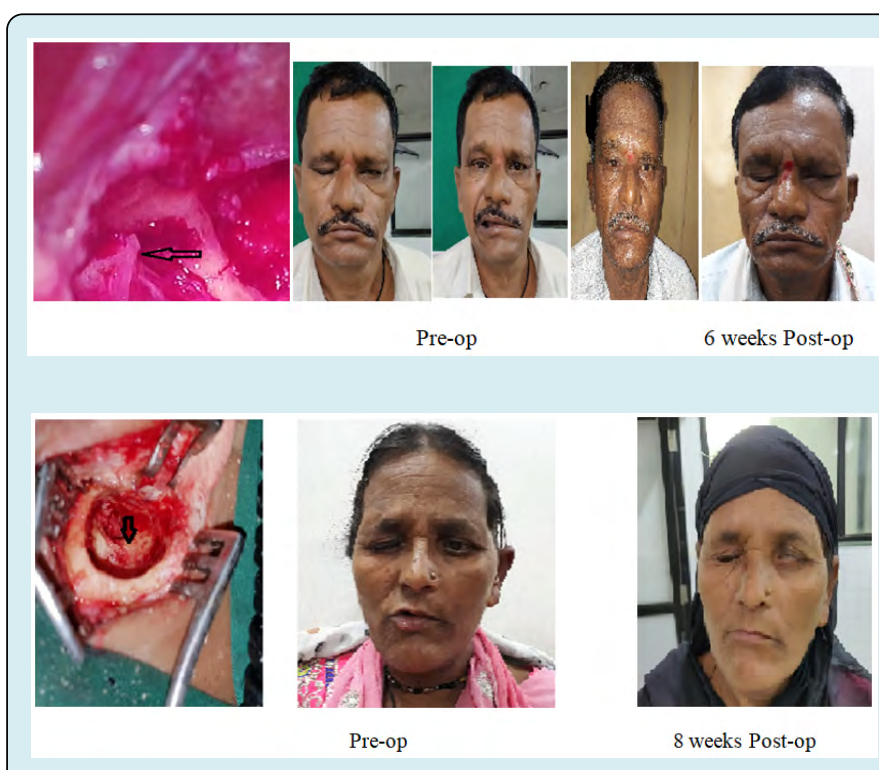

Figures 1 \& 2: Pre and Post-operative pictures of study subjects.

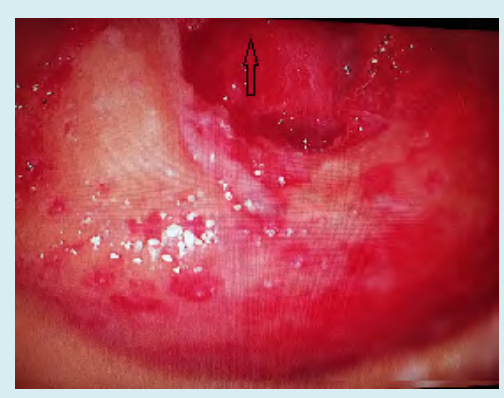

Figure 3.1: Intra-operatively lesion was at 1 st genu.

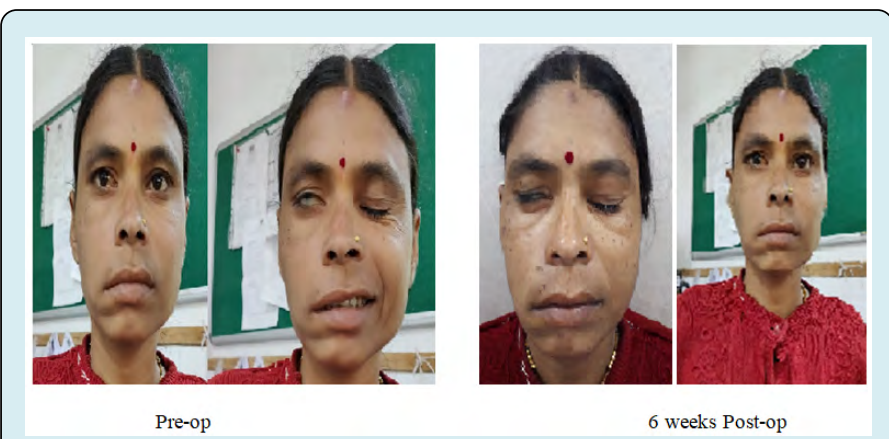

Figure 3.2: Pre and Post-operative pictures of study subject. 

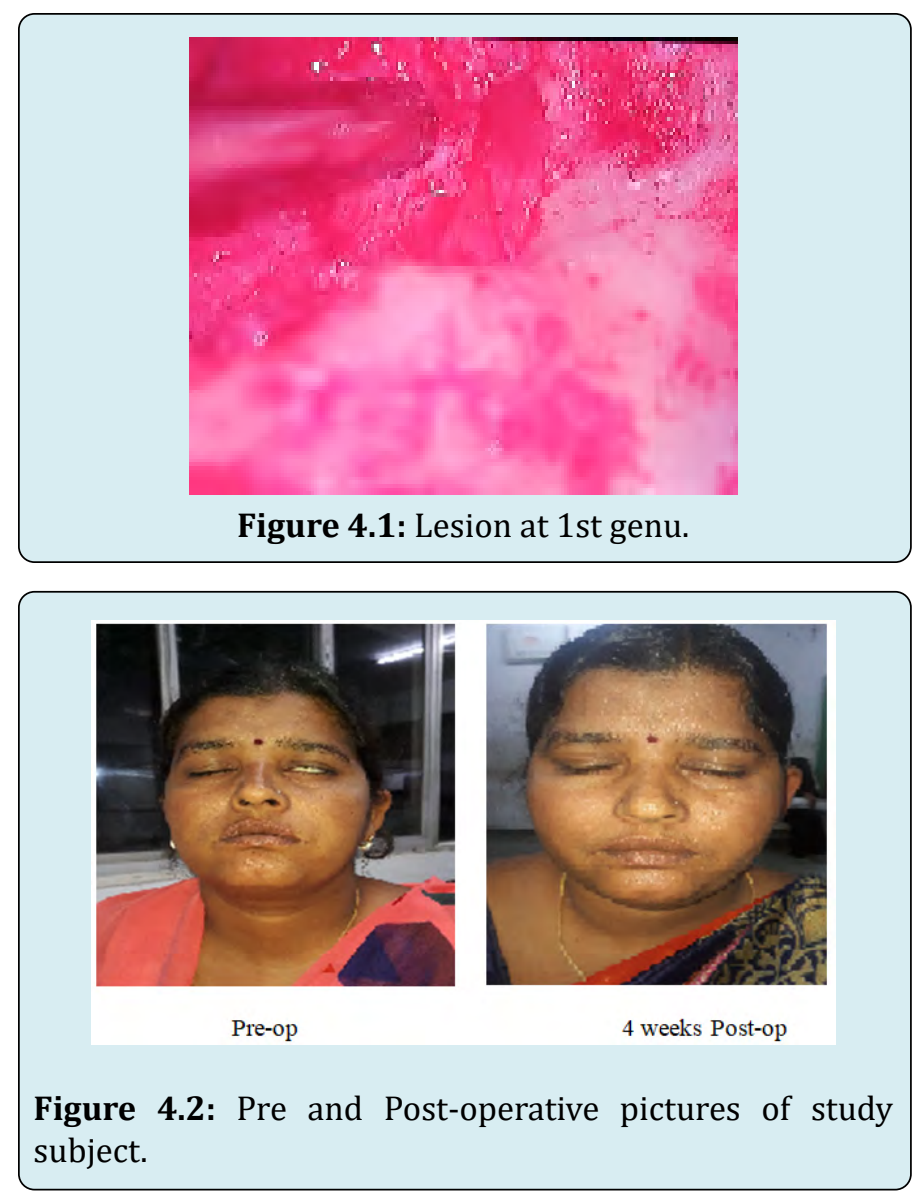

Figure 4.2: Pre and Post-operative pictures of study

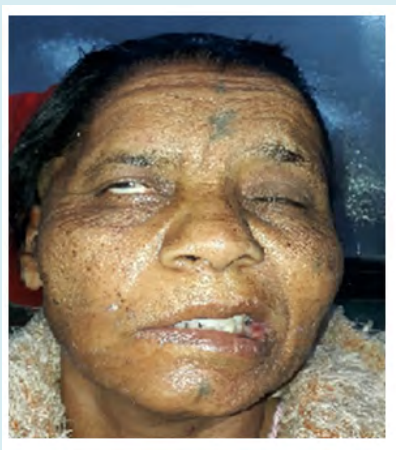

Pre-op

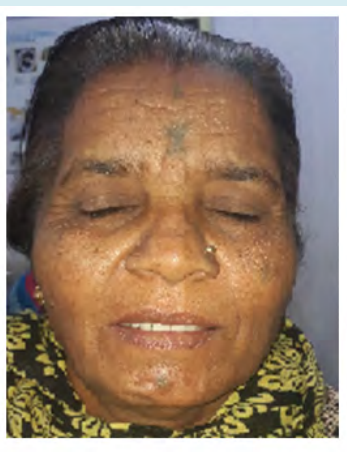

5 weeks Post-op
Figure 5: Pre \& Post-operative pictures of study subject.

\section{Observations and Results}

Our studied population included 8 males (40\%) and $12(60 \%)$ female patients. The mean age at diagnosis was $45.0( \pm 14.4)$ years. Among our group of patients, 2 (10\%) patients presented total facial paralysis (HB VI). Most common involved segment was tympanic segment of facial nerve.55\% of the patients from our study had lesion at tympanic segment followed by $30 \%$ patients had lesion at 1 st genu. Only $15 \%$ of patients had lesion at vertical segment and near stylomastoid foramen. Among these 5 $\%$ of the patients had complete transaction of the nerve at tympanic segment. Clinically patients were assesed to find out lesion of facial nerve before taking it for OT. In 35\% of patients, the lesion was at the level of 1st genu, the patient had U/ L hyperacusis, reduced salivation and loss of taste on ipsilateral 2/3 of tongue. Lacrimal function was intact. When lesion was at vertical segment of facial nerve, Ipsilateral taste sensation of anterior $2 / 3$ of tongue was intact, stapedial reflex lost and intact lacrimal function (Tables 1-3).

\begin{tabular}{|c|c|c|c|}
\hline Age group & \multicolumn{2}{|c|}{ Gender } & Total \\
\hline (In years) & Male (\%) & Female (\%) & (\%) \\
\hline $30-40$ & $2(10)$ & $2(10)$ & 20 \\
\hline $41-50$ & $3(15)$ & $6(30)$ & 45 \\
\hline $51-60$ & $1(5)$ & $3(15)$ & 20 \\
\hline$>61$ & $2(10)$ & $1(5)$ & 15 \\
\hline Total & $8(40)$ & $12(60)$ & $20(100 \%)$ \\
\hline
\end{tabular}

Table 1: Distribution of study subjects according to age and gender $(n=20)$.

All the patients had history of on and off ear discharge and ear pain but presented to us with the main concern of unilateral facial palsy which was acute.

\begin{tabular}{|c|c|c|c|}
\hline \multirow{2}{*}{ Clinical findings } & \multicolumn{2}{|c|}{ Gender } & Total \\
\cline { 2 - 4 } & $\begin{array}{c}\text { Male } \\
\text { (\%) }\end{array}$ & $\begin{array}{c}\text { Female } \\
\text { (\%) }\end{array}$ & (\%) \\
\hline $\begin{array}{c}\text { Cholesteatoma flakes } \\
\text { in ear }\end{array}$ & $2(10)$ & $6(30)$ & 40 \\
\hline $\begin{array}{c}\text { Granulation tissue in } \\
\text { ear }\end{array}$ & $3(15)$ & $4(20)$ & 35 \\
\hline $\begin{array}{c}\text { Severely retracted } \\
\text { tympanic membrane }\end{array}$ & $3(15)$ & $2(10)$ & 25 \\
\hline Total & $8(40)$ & $12(60)$ & $20(100 \%)$ \\
\hline
\end{tabular}

Table 2: Distribsution of study subjects according to clinical picture $(n=20)$.

\begin{tabular}{|c|c|c|c|}
\hline \multirow{2}{*}{$\begin{array}{c}\text { Grade of facial palsy } \\
\text { (pre -operatively) }\end{array}$} & \multicolumn{2}{|c|}{ Gender } & Total \\
\cline { 2 - 4 } & $\begin{array}{c}\text { Male } \\
\text { (\%) }\end{array}$ & $\begin{array}{c}\text { Female } \\
\text { (\%) }\end{array}$ & (\%) \\
\hline III & $2(10)$ & $6(30)$ & 40 \\
\hline IV & $4(20)$ & $2(10)$ & 30 \\
\hline V & $1(5)$ & $3(15)$ & 20 \\
\hline VI & $1(5)$ & $1(5)$ & 10 \\
\hline
\end{tabular}

Table 3: Distribution of study subjects according to HB grade of facial palsy at the time of presentation $(n=20)$. 


\section{Otolaryngology Open Access Journal}

Out of 20 subjects, most of the study subjects were having grade III facial palsy i.e.40\% followed by grade IV(30\%). The association between the grade of facial palsy and gender was not statistically significant.95\% of patients from the study improved with segmental facial nerve decompression along with mastoidectomy (irrespective of clinical presentation and site of lesion found intra-operatively). Only eradication of the disease by mastoidectomy was suggested by various authors in literature but segmental facial nerve decompression in these patients had additional benefit in view of clinical and aesthetic improvement and recovery as well as less requirement of post-operative steroids.

\section{Discussion}

There was always a controversy amongst the researchers regarding if the facial nerve decompression surgery to be done or not as there are many reported cases which improved spontaneously without any surgical intervention within 6 months of the presentation. However, there are many studies favouring facial nerve decompression. Quaranta, et al. studied 13 patients who underwent late decompression surgery for facial palsy reported HB-I and II recovery in $78 \%$ of patients [1]. Brodie and Thompson reviewed 58 facial nerve injuries and reported that all patients with incomplete paralysis in the beginning recovered and 8 out of 9 patients with delayed and 3 out of 5 patients with sudden onset facial paralysis recovered after surgical decompression. But 2 of those with immediate-onset complete paralysis presented poor prognosis [2]. Selection of surgical approach is determined by the site of the facial nerve injury and hearing status in the affected ear. Trans-mastoid approach, Middle cranial fossa approach, Trans-labyrinthine approach or else transmastoid supralabyrinthine approach. In the trans-mastoid approach, it enables mastoidectomy with posterior hypotympanotomy, it is simple for inexperienced surgeons and easy to expose the distal half of tympanic segment of facial nerve and horizontal segment and the same was used. Intra-operatively, there was bulky and edematous nerve at the site of lesion; for which we incised the epineurium of the nerve in that particular nerve segment and few millimeters proximal and distal to it. As the patients were diabetic, there was more edema of affected nerve segment along with granulation tissue all around the stapes footplate and hypotymapnum (in maximum number of patients).Usually, good reconstructive results for facial nerve repair were reported by Terzis, et al. even when a comparatively small number of axons were regenerated [3]. Facial nerve decompression is performed in severe cases when patient is at high risk of permanent total palsy and have a poor prognosis without any surgical/ aggressive intervention [3]. Unlike many previous studies, we performed segmental facial nerve decompression in patients which additionally benefited the diabetic subjects in recovery and reducing need for post-operative administration of steroids to reduce oedema. (Steroids were given only intra-operatively and 1 dose on post-op day1) Complications may be further surgical trauma to facial nerve, diminished hearing, vertigo, CSF leak, surgical site infection. Repair of facial nerve is contraindicated when the motor endplate muscle unit is not functional anymore [4]. Electrophysiological tests help to determine the functional status of the same. Gantz, et al. found greater recovery rates to grade I-II from facial palsy if decompression was done within 14 days of Bell's palsy [5]. CSOM causing facial palsy is most of the times due to cholesteatoma [6]. We found a very good results and improvement of $62.5 \%$ of males and $6.6 \%$ of studied female patients to HB grade- I on immediate post-op, 1 month and 6 month follow up. Generally, bony dehiscence of tympanic segment is seen in almost $33 \%$ of cases during middle ear surgery with cholesteatoma and is mostly at the tympanic portion of facial nerve found in the literature [7]. Savic and Djeric reported complete recovery in $70 \%$, partial in $24 \%$ and failure in $6 \%$ of cases. 19 following decompression surgery [8]. Cawthorne postulated in his study that nerve sheath incision should be performed for cases of complete paralysis [9].

\section{Conclusion}

Early decompression is beneficial to facial nerve. So it is better to release pressure over the nerve whenever needed and not to wait and watch .Our study supports segmental decompression of nerve irrespective of grade (severity) of facial nerve status at the time of presentation. It is found that diabetic patients are more prone for facial nerve paralysis and there is delayed recovery of facial nerve function in diabetic patients. As all patients in the study were diabetic, there was relative contraindication for steroid use; but epineural incision of affected nerve segment reduced the need of postoperative steroid therapy (incision of nerve sheath to release the pressure helps in reducing postoperative oedema). Though there are many controversies regarding the approach towards facial palsy patients whether conservative or surgical management to carry out, only disease eradication is enough; our study retrospectively proved the efficiency of facial nerve decompression surgery in affected patients both clinically and aesthetically.

Acknowledgement: All procedures performed in this case report was in accordance with ethical standards of the institution. Informed consent was obtained from all individual participants included in study.

\section{References}

1. Quaranta A, Campobasso G, Piazza F, Quaranta N, Salonna I (2001) Facial Nerve Paralysis in Temporal Bone Fractures: Outcomes after Late Decompression 


\section{Otolaryngology Open Access Journal}

Surgery. Acta Otolaryngol 121(5): 652-655.

2. Brodie HA, Thompson TC (1997) Management of complications from 820 temporal bone fractures. Am J Otol 18(2): 188-197.

3. Terzis JK, Wang W, Zhao Y (2009) Effect of Axonal Load on the Functional and Aesthetic Outcomes of the CrossFacial Nerve Graft Procedure for Facial Reanimation. Plastic and Reconstructive Surgery 124(5): 1499-1512.

4. Ho T, Byrne P, Hilinski JM, Hilger P (2018) Facial Nerve Repair.

5. Gantz BJ, Rubistein JT, Gidley P, Woodworth GG (1999) Surgical Management of Bell's palsy. Laryngoscope 109(8): 1177-1188.
6. Takeda T, Takebayashi S, Kakigi A, Nakatani H, Hamada $M$ (2009) Total decompression of the facial nervesuperior prelabyrinthine cell tracts approach. ORL J Otorhinolaryngol Relat Spec 71(1): 112-115.

7. Selesnik SH, Lynn Macrae AG (2001) The incidence of facial nerve dehiscence at surgery for cholesteatoma. Otol Neurotol 22(2): 129-132.

8. Savic DL, Djeric DR (1989) Facial paralysis in chronic suppurative otitis media. Clin Otolaryngol 14(6): 515517.

9. Cawthorne T (1969) Intratemporal facial palsy. Arch Otolaryngol 90(6): 789-799. 